\title{
Activities and Programs for Students with Special Needs
}

\author{
Jean-Philippe Drouhard
}

\section{Scope and Aims of the TSG 4}

Around the world, a considerable number of primary and secondary teachers are involved in teaching mathematics to special educational needs learners ("SEN-L") and a fair proportion of teacher educators are involved in preparing these teachers. But both, teachers and educators, very often are working under somewhat isolated circumstances. They are isolated geographically-it is not always easy to identify others working with SEN-L regionally, let alone nationally or internationally. And they are also isolated in terms of particular focus - specialists working with blind students, for example, may have little professional contact, if any, with specialists in the education of deaf students and those of Down's syndrome. Professional groups tend to be based more on the nature of the special needs of the students rather than on the learning of mathematics. This means that in the dialogue amongst educators concerned with SEN-L, mathematics education is hardly ever at centre stage. On the other hand, mathematics education researchers and teachers seldom have the specific knowledge about SEN-L. Mathematics educators do consider what mathematics for all should be, but the "all" rarely include SEN-L. Issues related to the mathematics education of students with special educational needs are currently under represented in the research community. What seems to be lacking is a community of mathematics educators dedicated to exploring this domain. Hence, there is a need to create common references and shared resources (in particular in

Organizers Co-chairs: Jean-Philippe DROUHARD (France) and Sung-Kyu CHOI; Team Members: Heloiza BARBOSA, Petra SCHERER, Jacinthe GIROUX; Liaison IPC Member: Bernard HODGSON.

J.-P. Drouhard $(\bowtie)$

Universidad de Buenos Aires, Buenos Aires, Argentina

e-mail: jpdrouhard@ccpems.exactas.uba.ar

(C) The Author(s) 2015

S.J. Cho (ed.), The Proceedings of the 12th International Congress

on Mathematical Education, DOI 10.1007/978-3-319-12688-3_29 
the case of inclusive education). In short, there is a strong need for a common culture of mathematics education for students with special educational needs.

At the end of the meeting it was agreed to create some kind of common Internet platform in order to communicate about how to start such a web of research and shared experience on special educational needs learners. A website (at the moment under construction) has been opened: https://sites.google.com/site/m4senl/ Contact: maths4senl@gmail.com.

What could Mathematics Education gain from the establishment of such common references and resources? First, mathematics education could become more significant in the lives of many students. There is a large number of young people and adult students for whom mathematics teaching may be "secondary" because the focus of their education is elsewhere. Second, insights developed in research with SEN-S could benefit mainstream mathematics teaching, through a re-analyses of assumptions about how mathematics is learned and what specific assessments tell us about students' abilities. Third, SEN-S may show unexpected dissociations between different aspects of mathematical knowledge. It is possible to find, for example, exceptional computational skills with little understanding of their conceptual basis in autistic. Finally, the discussion of different sorts of curricula with different resources appropriate for mathematics teaching while keeping mathematics as the focus of the discussion could lead to more diversified approaches to mathematics education.

\title{
Abstracts of the Communications and Posters Presented Within the TSG Meeting
}

\author{
Renato MARCONE, Miriam GODOY PENTEADO ${ }^{1}$ : A blind student at the uni- \\ versity: Challenges for mathematics teachers.
}

This presentation is based on the story of Mara, a student who became blind during a mathematics undergraduate course. The information for this case were obtained from interviews with Mara, her mother, university staff, colleagues and her teachers. As no blind student had ever before been at the mathematics faculty in question, the case of Mara took everyone by surprise. The first reaction from teachers was that Mara should take another subject-mathematics would be too difficult. However, given that Mara did not change her mind, the university staff had to define actions that would allow her to continue studying. In the article are presented more details of teachers' approaches. This case gives evidence of the challenge to be faced and possibilities that can be considered for teaching mathematics for students with special needs at the university.

\footnotetext{
1 marcone.renato@gmail.com, mirgps@gmail.com
} 
Solange FERNANDES ${ }^{2}$, Lulu HEALY: Representations of three-dimensional forms constructed by blind students: Relations between "seeing" and the "knowing".

The aim of this paper is to analyse how blind learners manage the conflicts between "seeing" and "knowing" in relation to two-dimensional representations of two geometric solids (a cube and a square-based pyramid). It seeks to locate elements within their interactions which make up the repertoires of "knowing" of those who do not see with their eyes, treating the processes involved in such interactions as acts of perception, with their origins in the body, and which serve a mediating role between environment, culture and brain.

Juliane LEUDERS ${ }^{3}$ : Auditory representations for blind and sighted students. Research into special education teacher education and professional development is sparse. This study set out to investigate factors that support special education teachers' ability to teach students with special needs fraction ideas. Working with three teachers in high school settings, the year long investigation into teacher professional development identified a number of key factors that contribute to student misconceptions and what teachers can do to mediate their learning difficulties.

Teresa ASSUDE, ${ }^{4}$ Jean-Philippe DROUHARD: Mathematics teaching situations with deaf or hard of hearing pupils.

This article aims to study some mathematics teaching situations which are proposed to the deaf or hard-of-hearing pupils in primary classroom for school inclusion (specialized classroom). We analyse some situations and identify some pupils' difficulties. Then we discuss the problem of the specificity or not of these teaching situations.

Rumiati RUMIATI, Robert J. WRIGHT ${ }^{5}$ : Research on number knowledge of students with Down syndrome: An experience from Indonesia.

This chapter presents the results of a small scale research study on the number knowledge of students with Down syndrome in Indonesia. Five students with Down Syndrome and ages ranging from 7 to 19 years, from a special education school in Yogyakarta city were interviewed to document their abilities in identifying numerals, solving number problems involving the use of unscreened and screened collections of counters, and solving one-digit and two-digit number problems in horizontal format. The approach and the schedule of assessment tasks in the interview were adapted from that used in Mathematics Recovery. The interviews were conducted individually and videotaped in order to capture subtle clues related to students' abilities. The number knowledge of the five students with Down syndrome is described, compared and discussed.

\footnotetext{
2 solangehf@gmail.com, lulu@baquara.com

3 juliane.leuders@ph-freiburg.de.

4 teresa.dos-reis-assude@univ-amu.fr.

5 rumiati1@yahoo.co.id, bob.wright@scu.edu.au.
} 
KOTAGIRI Tadato $^{6}$ : Mathematical achievement and creativity inherent in children with special needs.

The assessment of children's mathematical learning achievement entails recognition of the child's human rights to learn Basic Mathematics: (1) to be able to fulfil his/ her potential, and more importantly, (2) to be prepared for creative participation in his/her community, both in work and in other activities. Nonetheless, because many children with Special Needs face severe difficulties in obtaining the Basic Mathematical understanding and skills which they both deserve and need, they are effectively being denied their basic educational rights. This paper, based on years of using a clinical approach to remedial education, provides evidence of such children's remarkable possibilities for the achievement of Basic Mathematics, in particular exposing instances of significant creative response.

Marjolijn PELTENBURG, Marja VAN DEN HEUVEL-PANHUIZEN, Alexander $\mathrm{ROBITZSCH}^{7}$ : Yes, I got them all? Special education students' ability to solve ICT-based combinatorics problems.

This present study is aimed at revealing special education students' mathematical potential by means of a dynamic ICT-based assessment. The topic of investigation is elementary combinatorics, which is generally not taught in primary special education. Six combinatorics problems on finding all possible combinations of a number of different types of clothing items were presented on screen. Data were collected on students' performance in solving these items. The performances of students in regular education served as a reference. The total sample consisted of 84 students (8- to 13-year-olds) from special education and 76 students (7- to 11-yearolds) from regular education. Their mathematics ability ranged from halfway grade 2 to halfway grade 5 . The results showed that special education students are able to solve combinatorics problems equally successful as regular education students.

Pamela PAEK ${ }^{8}$ : Longitudinal analyses of students with special education needs in the United States on high-stakes mathematics assessments.

This paper analyzes one state's large-scale assessment (LSA) mathematics data over eight years in the United States, to identify patterns of progress and attrition rates for students with special education needs (SEN-S). A previous study (Paek and Domaleski 2011) showed that SEN-S tended to have slower growth and lower mathematics achievement compared to general education students (GE-S) across grades and years. However, the majority of SEN-S had missing data across years, indicating that any longitudinal reports of SEN-S' achievement and growth are not generalizable. Findings indicate that the majority of SEN-S do not have LSA data for a single year, change the types of assessment forms they take from year-to-year, and are not promoted to the next grade level as often as GE-S. These results reveal

${ }^{6}$ kotagiri@edu.u-ryukyu.ac.jp.

7 M.Peltenburg@uu.nl, m.vandenheuvel@fi.uu.nl, robitzsch.alexander@googlemail.com.

8 ppaek@nciea.org 
why a significant amount of SEN-S' data is missing, and how assumptions about data to measure achievement and growth for SEN-S are currently not tenable.

Eugenie KESTEL, Helen FORGASZ ${ }^{9}$ : An investigation of a targeted intervention program delivered by personal Video-conferencing for primary and middle school students with mathematical learning difficulties.

This paper describes an ongoing study investigating the effectiveness of an individual, conceptual instruction based, tuition program delivered by Personal VideoConferencing (PVC) for upper primary and middle school students with Mathematical Learning Difficulties (MLDs). The experimental intervention targets number sense and fluency with basic facts in mathematics. The effect of using a personal videoconferencing delivery modality on the mathematics anxiety levels experienced by students with MLDs is also investigated.

Rebecca SEAH: Mathematics professional development for special educators: Lessons learned from the field. ${ }^{10}$

Research into special education teacher education and professional development is sparse. This study set out to investigate factors that support special education teachers' ability to teach students with special needs fraction ideas. Working with three teachers in high school settings, the year long investigation into teacher professional development identified a number of key factors that contribute to student misconceptions and what teachers can do to mediate their learning difficulties.

\section{Leticia Pardo ${ }^{11}$ Special Education in Xalapa, Mexico: A brief history.}

The main focus of this work is to discuss briefly the history of Special Education services in Xalapa, capital city of the Mexican state named Veracruz. After 31 years serving this government office has experienced three phases of evolution: Integrated groups, Complementary Aid and Educational Integration. We recall some of the main characteristics of every one of these periods of time to explain the way that children with special needs were detected and how they were helped. One conclusion is that the philosophical base of Special Education has evolved from a kind of medical point of view to one based in social aspects.

Open Access This chapter is distributed under the terms of the Creative Commons Attribution Noncommercial License, which permits any noncommercial use, distribution, and reproduction in any medium, provided the original author(s) and source are credited.

9 eugenie.kestel@monash.edu, helen.forgasz@monash.edu

10 rtkseah@gmail.com.

11 rociopardo2000@yahoo.com.mx. 